\title{
Towards an Intra- and Interorganizational Perspective: Objectives and Areas of Activity of Digital Innovation Units
}

\author{
Jun-Patrick Raabe \\ Universität Hamburg \\ raabe@informatik.uni- \\ hamburg.de \\ Paul Drews \\ Leuphana Universität Lüneburg \\ paul.drews@leuphana.de
}

\author{
Bettina Horlach \\ Universität Hamburg \\ horlach@,informatik.uni- \\ hamburg.de
}

\author{
Ingrid Schirmer \\ Universität Hamburg \\ schirmer@informatik.uni- \\ hamburg.de
}

\begin{abstract}
Incumbent firms increasingly strive to embrace digital innovation, often via implementing dedicated digital innovation units (DIUs). As seizing the rapid and various digital innovation-related market movements may be overwhelming for an individual DIU, collaborations within ecosystems are perceived as crucial for continuously recognizing business opportunities and threats. Although this is a growing field of interest in recent research, insights into the objectives of DIUs and the consequent activities for effectively handling digital innovation are yet scarce. We address this issue by synthesizing 28 cases on DIUs through a qualitative meta-analysis. The analysis revealed that while DIUs enforce an intraorganizational cultural and overarching organizational design change, they also impose an interorganizational perspective with customeroriented digital expertise and innovation, as well as cultivation of digital innovation ecosystems. Thus, we contribute to the existing DIU research by clarifying these objectives and extending them to achieve a conscious interorganizational perspective with accompanying activities.
\end{abstract}

\section{Introduction}

With the advent of the digital age, in which digital technologies often shift the core of businesses [1, 2], firms understand the need to drive their own digital transformation [3]. Digital technologies have very prominent characteristics with important implications for firms' innovation management procedures [4]. "Digital innovation has radically changed the nature and structure of new products and services, spawned novel value creation and value appropriation pathways, enabled innovation collectives that involve dynamic sets of actors with diverse goals and capabilities, [...] and, more broadly, transformed entire industries in its wake" [5]. In the past, incumbent firms successfully sensed and responded to potential (digital) disruptions by (1) adapting their business models, (2) extending or updating to current digital technologies, or (3) sometimes cooperating with disruptors [6]. However, absorbing or integrating (digital) innovations still seems to be a challenging task for incumbent firms. To tackle these challenges, many incumbents have recently attempted to implement digital innovation units (DIUs). DIUs are described as dedicated organizational units that work with a high degree of freedom across firm boundaries and serve as enablers for embedding digital technologies into incumbent firms [7]. Their intent is to accelerate and trigger digital transformation $[7,8]$.

Prior research on DIUs has intraorganizationally focused on their design, distinct types, links to the main organization [7-9], and challenges that occur (e.g. due to missing objectives) [10]. Among these missing objectives, interorganizational aspects rarely seem to be addressed in the DIU literature, although the strong need for external collaboration is emphasized in digital innovation research [11]. As digital innovation causes a shift, especially in the locus of innovation, toward an intensified open and collaborative setting involving various partners [5], a digital innovation ecosystem perspective emphasizes a stronger focus on an incumbent firm's partners and its network. This specific ecosystem is defined as "a dynamic collective of interdependent actors and the resources they draw on to innovate with digital technology" [12].

By undertaking a focused study of DIU settings in practice, we strive to (1) obtain a deeper understanding of their objectives and areas of activity and (2) identify potential connections between DIUs and digital innovation ecosystems. By conducting a qualitative meta-analysis of previously published single and multiple case studies on DIUs, we strive to answer the following research questions: 
RQ1: What are the objectives and areas of activity of Digital Innovation Units?

RQ2: How is the interorganizational perspective addressed in Digital Innovation Units?

We answer these research questions by synthesizing five general objectives and seven areas of activity for DIUs. In this regard, we propose an extension of the DIU objectives stated by Fuchs et al. [8], in which the digital innovation ecosystem perspective is insufficiently addressed. The analysis of the cases reveals that the digital innovation ecosystem is very often addressed in DIUs. However, the interorganizational perspective is not sufficiently emphasized in DIU research and only superficially mentioned. With this paper, we seek to explore the DIU perspective and propose implications for management.

The remainder of this paper is structured as follows: Section 2 provides an overview of related research on DIUs and their connection to digital innovation ecosystems. Section 3 describes our research methodology. In Section 4, we introduce the synthesized DIU objectives and areas of activity. We discuss the results of our research in Section 5. Finally, we conclude with a summary, a discussion of limitations, and outlook in Section 6.

\section{Related research on DIUs}

Innovation and its management in firms are not new phenomena, as they have always been crucial for firms [13]. However, digital innovation differs significantly from prior non-digital innovation [14], which motivates firms to establish DIUs to accelerate their digital innovation endeavors [10]. In information systems research, authors have previously analyzed DIUs within the context of enabling (IT-)ambidexterity [15, 16], loose-tight-coupling [9] or bimodal IT [7], and established initial descriptive models about their characteristics and value contributions within firms. DIUs are seen as significant and fast accelerators for digital endeavors $[7,8,16,17]$. They are intended to "serve as [an] enabler for the integration of [digital innovation] into the main organization" [10]. Fuchs et al. [8] developed a taxonomy that addresses the objectives of DIUs by differentiating between "digital innovation", "cultural change", and "development of digital expertise". DIUs should foster an innovative culture, strengthen the digital expertise within the main organization, and/or implement digital solutions together with one or more business units [8]. By doing so, DIUs have to ensure that no current digital trends are missed, which could potentially be disruptive to the entire firm and harm day-to-day business. Differences exist between two [7,
17] or three DIU types $[9,16]$. These types are not mutually exclusive and may coexist within incumbent firms $[7,16]$. Despite strong similarities in their embedding, designs, or practices, differences are associated with the innovation type (process, product/service, business model [4]), the degree of innovation (incremental, radical, or disruptive focus $[18,19])$, the digital innovation stages [4] covered, or their market focus. In our prior research on DIUs [7], we separated DIU modes based on these differences. However, this intraorganizational view misses an interorganizational perspective 'across firm boundaries' and does not really consider ecosystems despite their high relevance in digital innovation research.

The ecosystem concept is of increasing significance within the field of managing technology and innovation [20]. This is highlighted by numerous recent calls for papers (e.g. MIS Quarterly 2019, ECIS 2020, ICIS 2020, HICSS 2021) [21]. This explosion of interest has resulted in a broad range of different (sub-)types and peculiarities of ecosystems causing a plethora of sometimes competing definitions and descriptions (e.g. business, platform, service, software, or innovation ecosystems [21]) [22]. The concept analyzes organic networks based on both positive and negative aspects (e.g. ecosystem-level competition, predation, or destruction) [20]. Their business and non-business actors have different attributes, decision-making principles, or purposes, which can cause unintended results at the ecosystem level [20]. In addition to finding decision-making principles and behavioral chains that strongly affect the growth and decline of the ecosystem [20], other main objectives are generally innovation and, more generally, value creation [21]. We will especially focus on these 'other objectives' and further draw on the innovation ecosystem concept (and especially on its nascent subtype of digital innovation ecosystems), as it "has emerged as a promising approach in the literature on strategy, innovation and entrepreneurship" [22].

Digital innovation ecosystems draw on business ecosystems [23] and differ in terms of value, as business ecosystems primarily (though not explicitly) relate to value capture, and digital innovation ecosystems relate to value creation [22]. Value capture is defined as "the individual firm-level actualized profit-taking; that is, [the path] firms eventually pursue to reach their own competitive advantages and [...] reap related profits" [24]; value creation refers to "the collaborative processes and activities of creating value for customers and other stakeholders" [24]. Ecosystem leaders create opportunities for other actors and capture value by providing services or goods 
while contributing to the overall health of an ecosystem [6]. Digital innovation ecosystems emerge through the interplay of adaptation and exaptation and evolve through various phases $[6,25,26]$. "Whereas adaptation refers to features that develop for a specific function, $[\ldots]$ exaptation refers to features that are later found to be useful for unintended functions" [6]. Digital innovation ecosystems underlie various tensions that need to be addressed and balanced. They need to be simultaneously stable and evolvable [27, 28]. Stability assures that financial and human resource investments of complementors and customers can yield long term returns [27]. Evolvability refers to adequately adjust to changes in customer requirements or market shifts [27]. Other paradoxical tensions that characterize ecosystems are (1) standard-variety, (2) control-autonomy, and (3) collective-individual [27]. While these tensions are especially crucial for architecting or creating digital innovation ecosystems, Selander et al. [29] describe the participation within ecosystems and focus on non-focal actors: the ecosystem participants who are at the boundary of ecosystems. They argue that ecosystem participants should not rely on single ecosystems [29]. Their model of capability search and redeem suggests that "it is imperative that non-focal actors pursue a pluralistic strategy, operating across digital [innovation] ecosystems and avoiding investing all efforts in the same ecosystem" [29]. Eaton et al. [30] emphasize the complex interplay of (non-)focal actors within ecosystems. They claim that these actors familiarize themselves with the technology and make independent decisions, which in turn may influence others' [30].

Researchers have compared digital innovation ecosystems to other ecosystem types, analyzed designs and domains, introduced strategies for adaptation and exaptation for focal and non-focal actors, or studied their evolution (e.g. $[6,12,22,25-27,29,30]$ ). However, a connection to DIUs has rarely been explored thus far. It has only been introduced by Svahn et al. [11] who studied the establishment of a temporary DIU within Volvo Cars as a first step in developing expertise for cross-fertilization and to increase continuous and incremental product development. This initial step assisted the company in engaging in external collaboration (1) with new partners, (2) to build a dynamic aftermarket while maintaining internal collaboration for competitive advantage, and (3) to identify and access new revenue streams while preserving internal coordination of existing value chains [11]. Since this was the only information systems research paper we could find addressing both DIUs and an interorganizational ecosystem perspective, we consider this a research gap and thus strive to gain further insight through an exploration of DIU cases.

\section{Research methodology}

We followed Habersang et al.'s [31] qualitative meta-analysis (meta-synthesis) research design. "A qualitative meta-analysis is a research design for synthesizing primary qualitative data from case studies" [31]. It allows the refinement, extension, or generation of new theory by identifying recurrent patterns in the re-examined cases [31-33]. This relatively new design is ideal for addressing this research gap, while answering our research questions and "provid[ing] more robust, generalizable and comprehensive findings" [31]. "[Q]ualitative case studies provide rich, contextualized empirical descriptions of the dynamics of a single setting across multiple levels of analysis" [31]. Critics could claim that single case studies only represent one case and thus may not be representative and generalizable [ 31 , 32 , as they do not offer any generic conclusions for other cases [31, 34]. Synthesizing multiple cases enables us to analyze a broader range of objectives and areas of activity, which facilitates discovery of new potentials for DIUs. This approach is suitable because qualitative meta-analyses can generate new (inductive) theories that can build links between rich qualitative evidence and deductive mainstream research [32].

Our literature search for single and multiple case studies on DIUs was carried out in two stages. First, we considered six research articles based on our knowledge base, as these authors initially described DIUs as a nascent phenomenon: Barthel et al. [9], Fuchs et al. [8], Holotiuk \& Beimborn [15], Jöhnk et al. [16], Raabe et al. [7], and Svahn et al. [11]. Second, we screened for relevant peer-reviewed articles within the AIS eLibrary, ACM Digital Library, and EBSCOhost Business Source Complete on 06-01-2020. Our search query included the following terms: 'digital innovation unit*', 'digital innovation

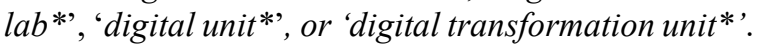
At least one of the terms needed to be included within the title, abstract or full text. We did not differentiate between research-in-progress and full papers, as long as sufficient data on the DIU cases were described (e.g. Barthel et al. [35]). After conducting a backward search, our data pool included 20 articles.

As described by Hoon et al. [32] and Rauch et al. [33], we defined criteria for inclusion and exclusion, rigorously ensuring the quality of our final data pool. We selected and included single and multiple case studies based on the following criteria of relevance. First, we only included case studies, which were 
described in depth (e.g. within the methodology section or within a dedicated section) and followed a systematic approach (e.g. Eisenhardt [36] or Yin [37]). Second, the described cases needed to match our general definition of DIUs ('established to accelerate digital transformation'; 'dedicated organizational units that work with a high degree of freedom across firm boundaries and serve as enablers for embedding digital technologies into their incumbent firms'). Third, the cases needed to include information about all the following dimensions: (1) main objectives \& areas of activity, (2) innovation orientation, (3) market focus of the innovation, (4) staffing, (5) importance of external partners, and (6) their governance \& structures, as described in the taxonomy of digital units by Fuchs et al. [8]. We excluded cases that were solely responsible for providing and maintaining IT services (e.g. Paletti's [38] case of 'Online at TfL'), as these are common tasks of a regular IT function ('service provider' [39]) and thus, do not fit our definition of DIUs.

In total, we included 14 articles with 28 mentioned cases on DIUs and their areas of activity in this study. We also included works by authors (Barthel et al. [9], Göbeler et al. [17], Jöhnk et al. [16], and Raabe et al. [7]) who used their empirical findings to describe good/best practices or types with detailed information about the areas of activity of the DIUs. Thus, the number of good/best practices or types of DIUs described is 10 . The cases are located in different countries and represent multiple industries. Therefore, they provide a good overview of established DIUs (Table 2 lists all cases, their areas of activity, and categorized objectives).

We analyzed and synthesized the areas of activity in three steps. In the first step, we followed the inductive category development approach discussed by Mayring [40] to identify all described areas of activity within the cases. Inductive or open coding is an interpretive process and helped us to gain new insights "by breaking through standard ways of thinking about or interpreting phenomena reflected in the data" [41]. In the second step, we iteratively clustered and synthesized the areas of activity, until the final set emerged. In the third step, we classified, if possible, the final areas of activity into the objectives described by Fuchs et al. [8]: digital innovation, cultural change, or digital expertise. For our data analysis, we used the MAXQDA software program.

\section{Results}

Based on our data, we were able to identify and synthesize seven final areas of activity. Most of these areas fit well within Fuchs et al.'s [8] objectives. To address the areas of activity that did not fit within the established objectives, we included two other dimensions: "Organizational Design Change" and "Digital Innovation Ecosystem". We think it is crucial to separate and subdivide the objectives, as these may have different impacts on the main organization.

"Organizational Design Change" dictates that DIUs should initiate and enable the realization of various organizational concepts: contextual [16], structural [17], or temporal ambidexterity [15], or a structural IT fast lane for digital endeavors (i.e., bimodal, trimodal, or multimodal IT [10]). Triggering organizational change requires high levels of responsibility and authority. Thus, this objective has a strategic and major impact on the main organization.

The objective "Digital Innovation Ecosystem" entails strengthening the ecosystem perspective and refers to the exploration of digital technologies and their ecosystems. This includes participation and cooperation within existing ecosystems and may result in creating new ones. This objective assists in protecting day-to-day business and has significant influence on the other objectives Digital Innovation and Digital Expertise, as digital technologies are selected based on the ecosystem and digital expertise needs to be accumulated for successful integration.

Table 1 lists the areas of activities, their classification within the objectives within the cases. An ' $x$ ' indicates the overlying objective, and an ' $(x)$ ' signals an indirect connection. Table 2 lists all DIU cases with their area(s) of activities. The order has no significance. The cases were arranged alphabetically (based on the authors). We included the authors, the

Table 1. Areas of activity and objectives

\begin{tabular}{|c|c|c|c|c|c|c|}
\hline \multirow{2}{*}{ No. } & \multirow{2}{*}{ Areas of Activity } & \multicolumn{5}{|c|}{ Objectives $^{1}$} \\
\hline & & DI & $\mathrm{CC}$ & $\mathrm{DE}$ & ODC & DIE \\
\hline$\# 1$ & Explore new digital technology trends: evaluate their strategic fit with the business & $\mathrm{x}$ & & & & $\mathrm{x}$ \\
\hline$\# 2$ & Discover, develop, implement, and diffuse digital solutions & $\mathrm{x}$ & & & & $(\mathrm{x})$ \\
\hline$\# 3$ & Foster a "Digital Culture" & & $\mathrm{x}$ & & (x) & \\
\hline$\# 4$ & Develop and leverage digital expertise (and agile methods) & & $(\mathrm{x})$ & $\mathrm{x}$ & $(\mathrm{x})$ & $(\mathrm{x})$ \\
\hline$\# 5$ & Participate and cooperate in existing digital innovation ecosystem(s): & $(\mathrm{x})$ & & $(\mathrm{x})$ & & $\mathrm{x}$ \\
\hline \#6 & Build (complimentary) digital innovation ecosystem(s) & $\mathrm{x}$ & & $(\mathrm{x})$ & & $\mathrm{x}$ \\
\hline \#7 & Enable organizational designs/concepts (suitable for fast innovation integration) & & $(\mathrm{x})$ & $(\mathrm{x})$ & $\mathrm{x}$ & \\
\hline
\end{tabular}

${ }^{1}$ DI = Digital Innovation; CC = Cultural Change; DE = Digital Expertise; ODC = Organizational Design Change; DIE = Digital Innovation Ecosystem 
Table 2. Identified DIU cases and their areas of activity

\begin{tabular}{|c|c|c|c|c|c|c|c|c|}
\hline \multirow[b]{2}{*}{ Author/s } & \multirow{2}{*}{$\begin{array}{c}\text { DIUs \& Industry } \\
\text { Case ID (C\#) / Type ID (T\#) }\end{array}$} & \multicolumn{7}{|c|}{ Areas of Activity (see Table 1) } \\
\hline & & $\# 1$ & $\# 2$ & $\# \mathbf{3}$ & \#4 & $\# 5$ & $\# 6$ & $\# 7$ \\
\hline \multirow[t]{2}{*}{ Barthel et al. [35] } & C1: Chemicals & $\mathrm{x}$ & From Discovery to (internal and external) Diffusion & $\mathrm{x}$ & & & & \\
\hline & C2: Tools & $\mathrm{x}$ & From Discovery to (internal and external) Diffusion & & & & $\mathrm{x}$ & \\
\hline Chanias [42] & C3: Finance & & & & $\mathrm{x}$ & $\mathrm{x}$ & & $\mathrm{x}$ \\
\hline Dremel et al. [43] & C4: Car & & From Development to (internal) Diffusion & $\mathrm{x}$ & $\mathrm{x}$ & & & \\
\hline \multirow[t]{5}{*}{ Fuchs et al. [8] } & C5: Steel & & From Discovery to (external) Diffusion & $\mathrm{x}$ & $\mathrm{x}$ & & & \\
\hline & C6: Food & & From Discovery to Pre-Development & $\mathrm{x}$ & $\mathrm{x}$ & & & \\
\hline & C7: Chemicals & & From Discovery to (internal and external) Diffusion & & $\mathrm{x}$ & $\mathrm{x}$ & & \\
\hline & C8: Investment & & From Discovery to (internal and external) Diffusion & $\mathrm{x}$ & $\mathrm{x}$ & & & \\
\hline & C9: Tools & & From Discovery to (internal and external) Diffusion & $\mathrm{x}$ & $\mathrm{x}$ & & & \\
\hline Gimpel et al. [44] & C10: Optics & & From Discovery to Pre-Development & & $\mathrm{x}$ & & & \\
\hline \multirow{9}{*}{$\begin{array}{l}\text { Holotiuk \& } \\
\text { Beimborn [15] }\end{array}$} & C11: Insurance & & From Discovery to (internal and external) Diffusion & & & & & $\mathrm{x}$ \\
\hline & C12: Transport & $\mathrm{x}$ & From Discovery to (internal and external) Diffusion & & & & & $\mathrm{x}$ \\
\hline & C13: Banking & & From Discovery to (internal and external) Diffusion & & & & & $\mathrm{x}$ \\
\hline & C14: Logistics & $\mathrm{x}$ & From Discovery to (internal and external) Diffusion & & & & & $\mathrm{x}$ \\
\hline & C15: Logistics & & From Discovery to (internal and external) Diffusion & & & & & $\mathrm{x}$ \\
\hline & C16: Banking & & From Discovery to (internal and external) Diffusion & $\mathrm{x}$ & & $\mathrm{x}$ & & $\mathrm{x}$ \\
\hline & C17: Apparel & & From Discovery to (internal and external) Diffusion & & & & & $\mathrm{x}$ \\
\hline & C18: Banking & & From Discovery to (internal and external) Diffusion & & & & & $\mathrm{x}$ \\
\hline & C19: Insurance & & From Discovery to (internal and external) Diffusion & $\mathrm{x}$ & & & & $\mathrm{x}$ \\
\hline Holotiuk [45] & C20: Banking & $\mathrm{x}$ & From Discovery to (internal) Diffusion & $\mathrm{x}$ & $\mathrm{x}$ & $\mathrm{x}$ & $\mathrm{x}$ & \\
\hline \multirow[t]{4}{*}{ Hund et al. [46] } & C21: Logistics & & From Acceleration to (internal) Diffusion & & & & & \\
\hline & C22: Credit & & From Acceleration to (internal) Diffusion & & & $\mathrm{x}$ & & \\
\hline & C23: Banking & & From Acceleration to (internal) Diffusion & & & & & \\
\hline & C24: Banking & $\mathrm{x}$ & From Acceleration to (internal) Diffusion & & & $\mathrm{x}$ & & \\
\hline $\begin{array}{l}\text { Rahrovani \& } \\
\text { Pinsonneault [47] }\end{array}$ & C25: Banking & & From Discovery to (external) Diffusion & & & & $\mathrm{x}$ & \\
\hline Svahn et al. [11] & C26: Car & $\mathrm{x}$ & & & $\mathrm{x}$ & $\mathrm{x}$ & & $\mathrm{x}$ \\
\hline Wulf et al. [48] & C27: Car & $\mathrm{x}$ & From Discovery to (internal) Diffusion & & $\mathrm{x}$ & & & \\
\hline $\begin{array}{l}\text { Zimmer (\& } \\
\text { Niemimaa) [49-51] }\end{array}$ & C28: Car & & From Discovery to (internal and external) Diffusion & $\mathrm{x}$ & $\mathrm{x}$ & & & $\mathrm{x}$ \\
\hline \multirow[t]{3}{*}{ Barthel et al. [9] } & MC, T1: Internal Facilitator & & From Discovery to (internal) Diffusion & & & & & \\
\hline & MC, T2: External Enhancer & & From Discovery to Pre-Development & & & & & \\
\hline & MC, T3: External Creator & & From Discovery to (external) Diffusion & & & $\mathrm{x}$ & & \\
\hline \multirow[t]{2}{*}{ Göbeler et al. [17] } & MC, T4: Active Engagement & $\mathrm{x}$ & From Development to (internal) Diffusion & $\mathrm{x}$ & $\mathrm{x}$ & & & \\
\hline & MC, T5: Passive Enablement & $\mathrm{x}$ & & $\mathrm{x}$ & $\mathrm{x}$ & & & $\mathrm{x}$ \\
\hline \multirow[t]{3}{*}{ Jöhnk et al. [16] } & SC, T6: Digital Unit & & From Discovery to (internal) Diffusion & & $\mathrm{x}$ & & & \\
\hline & SC, T7: Incubator & & From Discovery to (external) Diffusion & & & $\mathrm{x}$ & & \\
\hline & SC, T8: Cultural Change & & & $\mathrm{x}$ & $\mathrm{x}$ & & & \\
\hline \multirow[t]{2}{*}{ Raabe et al. [7] } & MC, T9: Coaching \& Screening & $\mathrm{x}$ & From Discovery to Pre-Development & $\mathrm{x}$ & $\mathrm{x}$ & $\mathrm{x}$ & & \\
\hline & MC, T10: Center of Excellence & & From Discovery to (internal) Diffusion and Impact & & & & & \\
\hline & $\sum$ & $\begin{array}{c}8 \\
(3) \\
\end{array}$ & $\begin{array}{l}26 \\
(8)\end{array}$ & $\begin{array}{l}10 \\
(4)\end{array}$ & $\begin{array}{l}12 \\
(5)\end{array}$ & $\begin{array}{c}7 \\
(3)\end{array}$ & $\begin{array}{c}3 \\
(0)\end{array}$ & $\begin{array}{l}12 \\
(1)\end{array}$ \\
\hline
\end{tabular}

cases, their industries, and the types. 'MC' stands for multiple case; 'SC' for single case and refers to whether the types are derived from one or multiple cases. The sum without brackets refers to the cases C1-C28; the sum within brackets stands for the types T1-T10. As almost all DIUs developed digital solutions, we further distinguished their areas of activity by digital innovation stages (inspired by Fichman et al. [4]). We differentiated between discovery, acceleration, pre-development (prototype status), development (minimum viable product / minimum awesome product status), (internal/external) diffusion, and impact measuring. We referenced the individual cases by their numeration (e.g. $\mathrm{C} 1$ refers to Barthel et al.'s [35] case). In the following, we will introduce and describe each area of activity in detail by giving examples from the cases.

\subsection{Explore new digital trends and evaluate their strategic fit with the incumbent firm \#1}

We identified 8 cases ( 3 types) of DIUs exploring new digital trends independently from the main organization. Within this area of activity, the exploration needs to be independent without prior influence from the main organization $(\mathrm{C} 12, \mathrm{C} 20, \mathrm{C} 24$, and T9). This is to ensure that DIUs are given the maximum degree of freedom to identify current digital trends that could disrupt markets. For example, C2 explores digital trends, evaluates and estimates possible future benefits, costs, and potential for scaling up the digital technology. Type T9 has a strong focus on trend screening, for which a dedicated team is responsible. 


\subsection{Discover, develop, implement, and diffuse digital solutions \#2}

Not surprisingly, almost all DIUs focus on digital solutions ( 26 cases, 8 types). As stated above, we made a further distinction by subdividing the areas of activity according to the digital innovation management stages. The discovery stage refers to the identification of (business) problems within the main organization (C14) or to the analysis of customer needs in order to solve their pain points $(\mathrm{C} 1$ and $\mathrm{C} 2)$. This is achieved either actively by generating ideas themselves (C4, T9, and T10) or passively by engaging employees and customers through appropriate techniques, such as idea/innovation pitches and other appropriate formats $(\mathrm{C} 10, \mathrm{C} 14, \mathrm{C} 16$, $\mathrm{C} 28$, and T9). In some cases (C21-24), discovery is not in a DIU's area of activity. These DIUs accelerate existing ideas from the main organization, so that they may eventually mature into an innovation. Predevelopment refers to the development of mockups or prototypes without further implementations. For example, DIU C9 hosts rapid-prototyping workshops in order to qualify and enable employees of the main organization to use agile working methods. Development refers to the implementation of a digital solution. The developed solutions vary and DIUs strive to focus on digital business processes (C16, $\mathrm{C} 20$, and $\mathrm{T} 1)$, products and services $(\mathrm{C} 4, \mathrm{C} 7, \mathrm{C} 9, \mathrm{C} 13$, $\mathrm{C} 18, \mathrm{C} 20, \mathrm{C} 27, \mathrm{~T} 6$, and T10), and business models (C1, C7-C9, C25, C28, and T7). Diffusion differs based on whether a DIU focuses on integrating their solutions into their main organization (internal, e.g. C21-24, T1, and T4) or/and diffuses it directly to the market (external, e.g. C5, T3, and T7). DIUs that integrate their solutions into the main organization (internal diffusion) tend to develop digital products, services, or processes. DIUs focusing on external diffusion strive to develop new digital business models for existing or potentially new customers (C25 and C28). The impact stage only received attention from type T10. It measures and monitors the impact of a digital technology that was implemented and integrated within its main organization.

\subsection{Foster a "digital culture" \#3}

We identified 10 DIUs (4 types) that strive to address cultural aspects. This includes enabling organizations to build an open culture of learningfrom-failure, encouraging employees to innovate, fostering a digital and agile mindset, and improving communication between employees $(\mathrm{C} 4-\mathrm{C} 6, \mathrm{C} 8, \mathrm{C} 9$, $\mathrm{C} 16, \mathrm{C} 19, \mathrm{C} 20, \mathrm{~T} 4, \mathrm{~T} 5$, and T8). For example, T8 is a dedicated unit that is explicitly focused on cultural change without addressing the development of digital solutions or exploring new digital trends.

\subsection{Developing and leveraging digital expertise and agile methods \#4}

We noted 12 DIU cases (5 types) within this area of activity. Developing and leveraging digital expertise and agile methods results in supporting or facilitating work within the main organization by providing digital capabilities, skills, tools, and IT infrastructure (T5). The development of new expertise can refer to new (agile) working methods, but also to technical skills in digital technologies. This involves leveraging core competencies (C1) or providing specific digital skills, for example in big data and predictive analytics (e.g. C4), by hosting workshops, hackathons, and/or open space initiatives (e.g. C10, $\mathrm{C} 28$, and T9). This either happens by qualifying current employees or by acquiring new digital talent. "A key measure was to bring experts from different areas of the holding company and the subsidiaries together to elaborate on concepts for an end-to-end digitization of customer journeys" [42] (C3). For example, DIU C4 strives to replace external consultancies and plans to improve their own expertise in specific digital trends to become interdependent. T5 aims to empower employees in the main organization to evaluate current trends. Thus, it is strongly connected to exploring new digital trends (\#1).

\subsection{Participate and cooperate in existing digital innovation ecosystem(s) \#5}

Although it is defined that DIUs work across firm boundaries [7], only 7 cases and 3 types name explicitly the participation and cooperation in ecosystems as a crucial area of activity. The DIUs addressing this area of activity focus especially on collaboration or networking with (potential) external partners ( $33, \mathrm{C} 16, \mathrm{C} 24, \mathrm{C} 26, \mathrm{~T} 3, \mathrm{~T} 7$, and $\mathrm{T} 9)$, acquisition of startups with a digital focus (T7), or investment in digital pioneers (T7). As stated earlier, C26 assists in collaborating with new partners and supports in building a dynamic aftermarket while maintaining internal collaboration [11]. This is strongly connected to building digital expertise, as it assists in the acquisition of digital talent (C3, C28).

\subsection{Build (complementary) digital innovation ecosystems(s) \#6}

Building (complementary) digital innovation ecosystems is closely related to the development of 
digital solutions (\#2). However, since external diffusion and impact measuring are hardly addressed, we have defined this area of activity separately to highlight the ecosystem perspective. The number of DIUs that address participation in ecosystems (\#5) explains the low number of DIUs covering the creation of new ecosystems. Only 3 cases focus on establishing and building new ecosystems, for example, by establishing new digital platforms to connect with partners and new sets of actors (C2, C20, and C25). For example, C20 strives "to provide a technological [digital innovation ecosystem], which allows [C20] to cooperate with different external partners and [...] offer digital services via [a]pplication [p]rogramming [i]nterfaces (APIs)" [45].

\subsection{Enable organizational designs \#7}

In this final area of activity, we identified the enablement of organizational designs or concepts that are suitable for fast digital innovation integration in 12 cases (1 type). Strongly connected to the objective of organizational design change, this area of activity refers to planning and designing organizational concepts, such as ambidexterity or *modal IT structures to ensure fast integration mechanisms for digital technologies. For example, T5 assists as a facilitator to initiate ambidextrous activities within the main organization. Like T5, C28 initiates structural ambidexterity by offering a single point of contact for orchestrating all digital transformation efforts and triggering design changes.

\section{Discussion}

This paper addresses the questions on the objectives and areas of activity and that the interorganizational ecosystem perspective is addressed in DIUs by conducting a meta-analysis of 28 DIU cases and 10 types. The results show that there is a link between DIUs and the digital innovation ecosystems mentioned in the cases, as collaboration with external partners and establishing networks is crucial for DIUs and their incumbent firms, as described by Svahn et al. [11]. Targeting the ecosystem is seen as an important point for DIUs within the cases. However, the importance of external collaboration is ignored in the DIU literature, as the intraorganizational perspective (especially the integration of digital technologies into incumbent firms) is focal. We therefore expanded the objectives of DIUs initially stated by Fuchs et al. [8] and introduced organizational design change and digital innovation ecosystem as new additional objectives of DIUs. We have ultimately identified seven areas of activity addressing both intra- and interorganizational perspectives.

Although many cases deal with the discovery stage of digital innovations, we were surprised that discovery did not refer to the identification or compilation of digital innovations on the market but the business problems of the main organization. This also explains the strong funding of business problemrelated ideas through specific initiatives and formats. As stated by Göbeler et al. [17], funding business problem-related ideas primarily assists on the promotion of incremental digital innovations and improves efficiency within the main organization. However, this sole perspective does not coincide with the initial intention of DIUs, as they are designed and established to accelerate especially radical and potentially disruptive digital innovations [7]. Although strong internal collaboration with the main organization is crucial [10], strong internal collaboration could be a hindrance. A business problem-based approach may force a limited perspective and put digital technologies and their ecosystems in the background. We therefore identified another approach currently pursued by DIUs: digital innovation-driven business change. These approaches are both pursued by DIUs and crucial in order to accelerate digital endeavors. In the following, we describe these two approaches as a "two-fold approach", as it has significant theoretical and managerial implications for DIUs.

\subsection{A two-fold approach for DIUs}

Business problem-based digital innovation selection refers to and is derived from the most commonly identified area of activity in the cases: "Discover, develop, implement, and diffuse digital solutions" (\#2). It has been previously described within Barthel et al.'s [9] ideal type of internal facilitator (T1) or Raabe et al.'s [7] practice of coaching \& screening type (T9). First, business problems are compiled. This occurs either actively by analyzing the main organization or passively by initializing open space initiatives or idea acceleration programs. Second, current digital technologies are explored and possible solutions to these problems are developed. Therefore, digital innovation ecosystems are searched for solutions. Business problem-based digital innovation selection may foster radical innovations, but the close connection to the main organization could harm innovation behavior. Hence, it assists primarily in accelerating incremental innovations.

Digital innovation-driven business change refers to a stronger need for highlighting digital innovation 
ecosystems, which is often neglected in the literature due to the stronger intraorganizational focus. Without being biased by incumbent firms, current digital technology trends are explored and evaluated as a first step. This ensures that particularly radical and potentially disruptive innovations are explored and evaluated. The size and set of actors are important indicators for evaluating the impact of a digital innovation. Thus, analyzing digital innovation ecosystems is crucial. Specifically targeting emerging ecosystems could attract young digital talent. Evaluating potential threats and risks is also crucial, as this may protect an incumbent firm's business model from being disrupted. In the event that a potentially disruptive digital innovation is detected, a DIU then makes recommendations on whether its incumbent firm has to adapt their business model, extend and update current technologies, or participate in emerging ecosystems and foster growth and internal disruption [6]. In this second step, a DIU requires a high degree of freedom in order to initiate (radical) change. Business change refers to both changing processes within the main organization and adapting business models.

\subsection{Navigating through ecosystems}

The cases revealed that digital innovation ecosystems are considered in DIUs. Thus, navigating through them and collaborating with external partners is key for DIUs. DIUs should not solely focus and participate in one but multiple ecosystems as non-focal actors [29]. Navigating through ecosystems does not only refer to participation and cooperation; it also includes the creation of new ecosystems as a result of this exploration. In terms of building ecosystems (e.g. as platform creator) DIUs should be aware of the described tensions that characterize ecosystems [27]. Figure 1 illustrates the two-fold approach. It should motivate both the intra- and interorganizational perspectives, in which the objective digital innovation ecosystem plays a major role. Digital innovation ecosystems affect the other objectives, as the development of digital solutions requires specific digital expertise and depends on the technologies screened, observed, and evaluated. Depending on the influence of a digital innovation, this may result in cultural and organizational design change. The solid and dotted arrows within a DIU from the objectives to the areas of activity represent the allocations from Table 1. These objectives do not necessarily have to be covered within one DIU; they can be structurally separated and managed through multiple concurrent initiatives [16].

\section{Conclusion and outlook}

We extended the objectives of DIUs by considering both the intra- and interorganizational perspectives. We formulated seven areas of activity that are currently pursued within DIUs. Not surprisingly, digital innovation is the most addressed objective. However, we noticed that DIUs pay a lot of attention to current business problems in developing solutions for their main organization to improve efficiency. This approach does not lead to the promotion of radical change, but rather incremental digital innovations, as already described by Göbeler et al. [17]. DIUs are encouraged to (1) analyze emerging ecosystems independently of the business, (2) participate and cooperate in them, or even (3) create new ones. The identified areas of activity highlight their status as a strategic unit to accelerate digital endeavors, protect day-to-day business, and bring significant change within incumbent firms by navigating through ecosystems. This article does not come without limitations. DIUs occur in various forms and have multifaceted names. Thus, other terms might need to be included for DIUs. Our qualitative metaanalysis includes DIU cases and types equally, although these types were derived empirically from other DIUs. Further research can be built on our results. We extended the objectives of DIUs and identified a link to digital innovation ecosystems. An overarching view that takes into account corporate and IT governance remains necessary. Such a perspective would explore methods of positioning DIUs within incumbent firms for effective and efficient digital

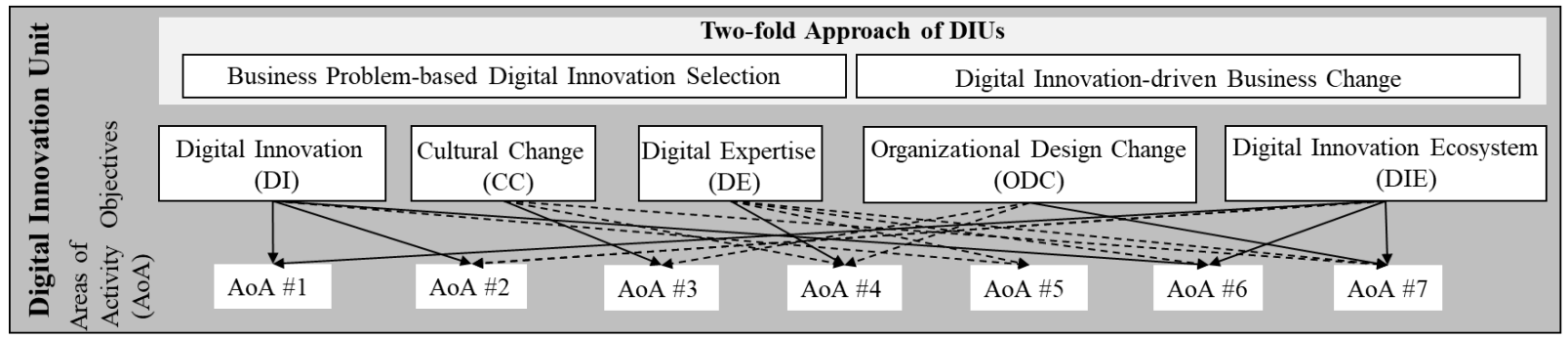

Figure 1. The two-fold approach, objectives, and areas of activity of DIUs 
innovation management. We have raised this issue in our prior articles $[7,10]$, but we did not deeply explore this area. An in-depth case study with firms that, for example, have created a digital innovation fast lane with DIUs might be appropriate. A longitudinal analysis of the evolution of DIUs is also currently missing. As there is a lot of "talk" of being digital-first, but perhaps not enough "walk", we find it intriguing to elaborate more on the aspect of instilling especially a digital culture in incumbent firms.

\section{References}

[1] Bharadwaj, A., O.A. El Sawy, P.A. Pavlou, and N. Venkatraman, "Digital Business Strategy: Toward a Next Generation of Insights", MIS Quarterly, 37(2), 2013, pp. $471-482$.

[2] Nissen, V. and A.v. Rennenkampff, "Measuring the Agility of the IT Application Systems Landscape", International Conference on Wirtschaftsinformatik (WI), 2017.

[3] Ross, J.W., C.M. Beath, and M. Mocker, Designed for digital: How to architect your business for sustained success, MIT Press, Cambridge, 2019.

[4] Fichman, R.G., B.L.D. Santos, and Z. Zheng, "Digital Innovation as a Fundamental and Powerful Concept in the Information Systems Curriculum", MIS Quarterly, 38(2), 2014, pp. 329-343.

[5] Nambisan, S., "Information Technology and Product/Service Innovation: A Brief Assessment and Some Suggestions for Future Research", Journal of the Association for Information Systems, 14(4), 2013, pp. 215-226.

[6] Beltagui, A., A. Rosli, and M. Candi, "Exaptation in a digital innovation ecosystem: The disruptive impacts of 3D printing", Research Policy, 49(1), 2020.

[7] Raabe, J.-P., B. Horlach, P. Drews, and I. Schirmer, "Digital Innovation Units: Exploring Types, Linking Mechanisms and Evolution Strategies in Bimodal IT Setups", International Conference on Wirtschaftsinformatik (WI), 2020.

[8] Fuchs, C., P. Barthel, I. Herberg, M. Berger, and T. Hess, "Characterizing Approaches to Digital Transformation: Development of a Taxonomy of Digital Units", International Conference on Wirtschaftsinformatik (WI), 2019.

[9] Barthel, P., C. Fuchs, B. Birner, and T. Hess, "Embedding Digital Innovations in Organizations: A Typology for Digital Innovation Units", International Conference on Wirtschaftsinformatik (WI), 2020.

[10] Raabe, J.-P., B. Horlach, I. Schirmer, and P. Drews, "'Forewarned is Forearmed': Overcoming Multifaceted Challenges of Digital Innovation Units", Americas Conference on Information Systems (AMCIS), 2020.
[11] Svahn, F., L. Mathiassen, and R. Lindgren, "Embracing Digital Innovation in Incumbent Firms: How Volvo Cars Managed Competing Concerns", MIS Quarterly, 41(1), 2017, pp. 239-253.

[12] Wang, P., "THEORIZING DIGITAL INNOVATION ECOSYSTEMS: A MULTILEVEL ECOLOGICAL FRAMEWORK", European Conference on Information Systems (ECIS), 2019.

[13] Chiesa, V., "Global R\&D Project Management and Organization: a Taxonomy", Journal of Product Innovation Management, 17(5), 2000, pp. 341-359.

[14] Yoo, Y., O. Henfridsson, and K. Lyytinen, "The New Organizing Logic of Digital Innovation: An Agenda for Information Systems Research", Information Systems Research, 21(4), 2010, pp. 724-735.

[15] Holotiuk, F. and D. Beimborn, "Temporal Ambidexterity: How Digital Innovation Labs Connect Exploration and Exploitation for Digital Innovation", International Conference on Information Systems (ICIS), 2019.

[16] Jöhnk, J., P. Ollig, S. Oesterle, and L.-N. Riedel, "The Complexity of Digital Transformation - Conceptualizing Multiple Concurrent Initiatives", International Conference on Wirtschaftsinformatik (WI), 2020.

[17] Göbeler, L., D. Schaar, and P. Hukal, "INITIATING AMBIDEXTERITY THROUGH DIGITAL INNOVATION LABS", European Conference on Information Systems (ECIS), 2020.

[18] Christensen, C.M., The innovator's dilemma: When new technologies cause great firms to fail, Harvard Business School, Boston, 1997.

[19] Dahlin, K.B. and D.M. Behrens, "When is an invention really radical?: Defining and measuring technological radicalness", Research Policy, 34(5), 2005, pp. 717-737.

[20] Tsujimoto, M., Y. Kajikawa, J. Tomita, and Y. Matsumoto, "A review of the ecosystem concept Towards coherent ecosystem design", Technological Forecasting and Social Change, 136, 2018, pp. 49-58.

[21] Guggenberger, T., F. Möller, T. Haarhaus, I. Gür, and B. Otto, "ECOSYSTEM TYPES IN INFORMATION SYSTEMS", European Conference on Information Systems (ECIS), 2020.

[22] Gomes, L.A.d.V., A.L.F. Facin, M.S. Salerno, and R.K. Ikenami, "Unpacking the innovation ecosystem construct: Evolution, gaps and trends", Technological Forecasting and Social Change, 136, 2018, pp. 30-48.

[23] Moore, J.F., "Predators and Prey: A New Ecology of Competition", Harvard Business Review, 1993.

[24] Ritala, P., V. Agouridas, D. Assimakopoulos, and O. Gies, "Value creation and capture mechanisms in innovation ecosystems: a comparative case study", International Journal of Technology Management, 63, 2013, p. 244. 
[25] Kim, D.D., B. Tan, Felix Ter Chian Tan, J. Ondrus, and J. Oh, "IS Capabilities in the Development of an Innovation Ecosystem: A Case Study of the Hallyu (Korean Wave) Phenomenon", International Conference on Information Systems (ICIS), 2017.

[26] Chae, B., "A General framework for studying the evolution of the digital innovation ecosystem: The case of big data", International Journal of Information Management, 45, 2019, pp. 83-94.

[27] Wareham, J., P.B. Fox, and J.L. Cano Giner, "Technology Ecosystem Governance", Organization Science, 25(4), 2014, pp. 1195-1215.

[28] Tilson, D., K. Lyytinen, and C. Sørensen, "Digital Infrastructures: The Missing IS Research Agenda", Information Systems Research, 21(4), 2010, pp. 748-759.

[29] Selander, L., O. Henfridsson, and F. Svahn, "Capability Search and Redeem across Digital Ecosystems", Journal of Information Technology, 28(3), 2013, pp. 183-197.

[30] Eaton, B., S. Elaluf-Calderwood, C. Sørensen, and Y. Yoo, "Distributed Tuning of Boundary Resources: The Case of Apple's iOS Service System", MIS Quarterly, 39(1), 2015, pp. 217-243.

[31] Habersang, S., J. Küberling-Jost, M. Reihlen, and C. Seckler, "A Process Perspective on Organizational Failure: A Qualitative Meta-Analysis", Journal of Management Studies, 56(1), 2019, pp. 19-56.

[32] Hoon, C., "Meta-Synthesis of Qualitative Case Studies", Organizational Research Methods, 16(4), 2013, pp. 522556.

[33] Rauch, A., R. van Doorn, and W. Hulsink, "A Qualitative Approach to Evidence-Based Entrepreneurship: Theoretical Considerations and an Example Involving Business Clusters", Entrepreneurship Theory and Practice, 38(2), 2014, pp. 333-368.

[34] Dyer, W.G. and A.L. Wilkins, "Better Stories, Not Better Constructs, To Generate Better Theory: A Rejoinder to Eisenhardt", Academy of Management Review, 16(3), 1991, pp. 613-619.

[35] Barthel, P., N. Stark, and T. Hess, "EXPLORING NEW AREAS FOR PROJECT PORTFOLIO MANAGEMENT - EVOLVING PRACTICES FOR DIGITAL TRANSFORMATION PROJECTS", European Conference on Information Systems (ECIS), 2020.

[36] Eisenhardt, K.M., "Building Theories from Case Study Research", Academy of Management Review, 14(4), 1989, pp. 532-550.

[37] Yin, R.K., Case study research: Design and methods, 5th edn., Sage Publication, London, 2014.

[38] Paletti, A., "How to manage ICTs mediated coproduction: a Public Value Perspective", Hawaii International Conference on System Sciences (HICSS), 2018.
[39] Guillemette and Paré, "Toward a New Theory of the Contribution of the IT Function in Organizations", MIS Quarterly, 36(2), 2012, p. 529.

[40] Mayring, P., Qualitative content analysis: theoretical foundation, basic procedures and software solution, Beltz, Klagenfurt, 2014.

[41] Corbin, J.M. and A. Strauss, "Grounded theory research: Procedures, canons, and evaluative criteria", Qualitative Sociology, 13(1), 1990, pp. 3-21.

[42] Chanias, S., "MASTERING DIGITAL TRANSFORMATION: THE PATH OF A FINANCIAL SERVICES PROVIDER TOWARDS A DIGITAL TRANSFORMATION STRATEGY", European Conference on Information Systems (ECIS), 2017.

[43] Dremel, C., M. Herterich, J. Wulf, J.-C. Waizmann, and W. Brenner, "How AUDI AG Established Big Data Analytics in Its Digital Transformation", MIS Quarterly Executive, 16(2), 2017.

[44] Gimpel, H., S. Hosseini, R. Huber, L. Probst, M. Röglinger, and U. Faisst, "Structuring Digital Transformation: A Framework of Action Fields and its Application at ZEISS", Journal of Information Technology Theory and Application (JITTA), 19(1), 2018.

[45] Holotiuk, F., "The Organizational Design of Digital Innovation Labs: Enabling Ambidexterity to Develop Digital Innovation", International Conference on Wirtschaftsinformatik (WI), 2020.

[46] Hund, A., F. Holotiuk, H.-T. Wagner, and D. Beimborn, "KNOWLEDGE MANAGEMENT IN THE DIGITAL ERA: HOW DIGITAL INNOVATION LABS FACILITATE KNOWLEDGE RECOMBINATION", European Conference on Information Systems (ECIS), 2019.

[47] Rahrovani, Y. and A. Pinsonneault, "Expertise Diversity, Knowledge Integration, and Team Innovation", International Conference on Information Systems (ICIS), 2017.

[48] Wulf, J., T. Mettler, and W. Brenner, "Using a Digital Services Capability Model to Assess Readiness for the Digital Consumer", MIS Quarterly Executive, 16(3), 2017.

[49] Zimmer, M. and M. Niemimaa, "Cultivating a 'Digital Jungle': Toward a Hybrid Governance Perspective on Infrastructure Evolution", Pacific Asia Conference on Information Systems (PACIS), 2020.

[50] Zimmer, M.P. and M. Niemimaa, "Navigating in the Digital Jungle: Articulating Combinatory Affordances of Digital Infrastructures for Collaboration", Pacific Asia Conference on Information Systems (PACIS), 2019.

[51] Zimmer, M., "Improvising Digital Transformation: Strategy Unfolding in Acts of Organizational Improvisation", Americas Conference on Information Systems (AMCIS), 2019. 